\title{
The role of cochlear implant positioning on MR imaging quality: a preclinical in vivo study with a novel implant magnet system
}

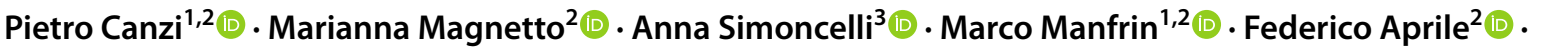

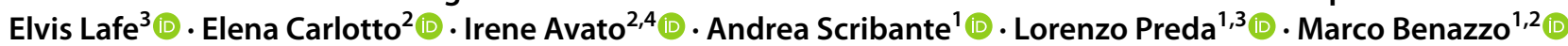

Received: 4 May 2021 / Accepted: 16 July 2021 / Published online: 9 August 2021

(c) The Author(s) 2021

\begin{abstract}
Purposes To investigate the effects for Ultra 3D cochlear implant (CI) positioning on MR imaging quality, looking at a comprehensive description of intracranial structures in cases of unilateral and bilateral CI placement.

Methods Four CI angular positions $\left(90^{\circ}, 120^{\circ}, 135^{\circ}\right.$ and $\left.160^{\circ}\right)$ at $9 \mathrm{~cm}$ distance from the outer-ear canal were explored. The 1.5 T MRI assessment included our institutional protocol for the investigation of brain pathologies without gadolinium application. Three investigators (two experienced neuroradiologists and one experienced otoneurosurgeon) independently evaluated the MR findings. A 4-point scale was adopted to describe 14 intracranial structures and to determine which CI positioning allowed the best image quality score and how bilateral CI placement modified MRI scan visibility.

Results A high positive correlation was found between the three blinded observers. Structures situated contralateral from the CI showed high-quality values in all four placements. Structures situated ipsilaterally provided results suitable for diagnostic purposes for at least one position. At $90^{\circ}$, artifacts mainly involved brain structures located cranially and anteriorly (e.g., temporal lobe); on the contrary, at $160^{\circ}$, artifacts mostly influenced the posterior fossa structures (e.g., occipital lobe). For the bilateral CI condition, MR imaging examination revealed additional artifacts involving all structures located close to either CI, where there was a signal void/distortion area.

Conclusions Suitable unilateral CI positioning can allow the visualization of intracranial structures with sufficient visibility for diagnostic purposes. Bilateral CI positioning significantly deteriorates the anatomical visibility. CI positioning might play a crucial role for patients who need post-operative MRI surveillance.
\end{abstract}

Keywords Cochlear implant $\cdot$ Implant positioning $\cdot$ MRI artifacts $\cdot$ Rotatable internal magnets $\cdot$ Diametrically bipolar magnets

Pietro Canzi

pietro.canzi@unipv.it

1 Department of Clinical, Surgical, Diagnostic and Pediatric Sciences, University of Pavia, Pavia, Italy

2 Department of Otorhinolaryngology, Fondazione IRCCS Policlinico San Matteo, University of Pavia, Viale Camillo Golgi, 19, 27100 Pavia, Italy

3 Department of Diagnostic Radiology and Interventional Radiology and Neuroradiology, Fondazione IRCCS Policlinico San Matteo, University of Pavia, Pavia, Italy

$4 \mathrm{PhD}$ in Experimental Medicine, University of Pavia, Pavia, Italy

\section{Introduction}

Over time, the MRI compatibility issue has become one of the most relevant challenges faced by years of cochlear implant (CI) technological research. Pain, magnet migration, reversal of magnet polarity are possible adverse events related to CI-MRI interaction [1]. To overcome these limitations, new generations of CIs with self-aligning magnets have been developed. In 2014, Med-El (Med-El, Innsbruck, Austria), first designed a CI model with a freely rotating magnet in one axis. More recently, Advanced Bionics AG (AB-Stäfa, Switzerland), released a new CI model (HiRes ${ }^{\mathrm{TM}}$ Ultra 3D) featuring four independent magnet bars free to rotate on two axes. Manufacturers' guidelines indicate MRI compatibility up to $3 \mathrm{~T}$ without the need for magnet removal or head bandaging [2]. Once those MRI 
safety issues have been satisfied, new considerations need to be addressed. Patients previously precluded from cochlear implantation, because of the need for ongoing MRI surveillance, may now be candidates for CI surgery. For these patients it is necessary to evaluate the possibility of visualizing the particular intracranial structures of interest. The depiction of anatomical structures mainly depends on the location and extent of signal void and distortion area produced by the interaction between the internal CI magnet system and the MR field. Viable strategies to handle artifact effects include internal magnet removal [3], head orientation [4, 5], MRI algorithms manipulation [6-8] and CI positioning [9]. Until now, only two studies (performed by the same team of researchers) have investigated the influence of CI positioning with non-rotatable internal magnets on MRI artifacts. Both studies, focused on posterior fossa visibility $[9,10]$. The aim of the present study was to investigate the effects of Ultra 3D CI position on MR imaging quality, looking at a comprehensive description of intracranial structures in the case of either unilateral or bilateral implantation.

\section{Materials and methods}

The study was conducted on two healthy adult male volunteers who offered written informed consent after approval from the institutional review board and medical ethics committees. Two AB HiRes ${ }^{\mathrm{TM}}$ Ultra 3D CIs with Slim $\mathrm{J}$ electrode arrays were supplied for research purposes. A thin single-layer medical gauze was tied to the volunteer's head. The implant package, with its magnet system in place, was then placed onto the head of each volunteer and held in place by a medical patch. No bandage, or polyethene block were used to restrain the implant package. The CI package's location was determined with respect to the nasion-outer-ear canal line, the package being oriented in this direction, with the center of the magnet located $9 \mathrm{~cm}$ behind the outer-ear canal. Four angles $\left(90^{\circ}, 120^{\circ}\right.$, $135^{\circ}$ and $160^{\circ}$ ) of orientation were explored (Fig. 1) for both unilateral and bilateral placement.
Fig. 1 CI positioning on healthy volunteer $\left(90^{\circ}, 120^{\circ}, 135^{\circ}\right.$, $\left.160^{\circ}\right)$
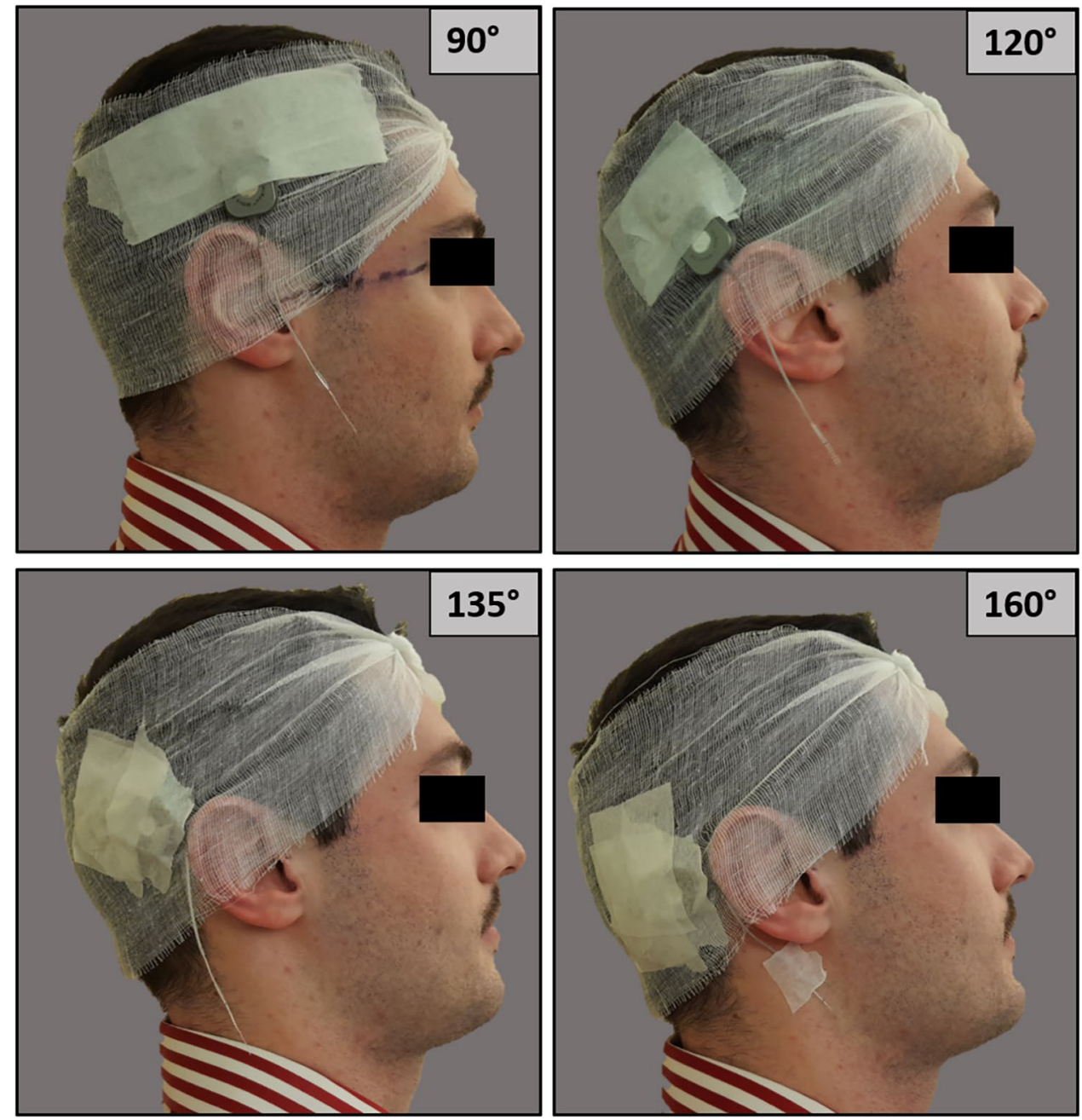


\section{Imaging study protocol}

All MRI examinations were carried out using the Ingenia ${ }^{\mathrm{TM}}$ (Philips Medical Systems, Best, Netherlands) 1.5 T MRI scanner. The MRI assessment included our institutional protocol (without gadolinium) for the investigation of brain pathologies. The acquisition protocol involved planar T1 and T2 weighted (w) turbo spin echo sequences. In particular, the following parameters were selected:

- Axial T1w: repetition time (TR) $550 \mathrm{~ms}$, echo time (TE) $10 \mathrm{~ms}$, slice thickness $2.5 \mathrm{~mm}$, field of view (FoV) $120 \times 179 \mathrm{~mm}^{2}$, acquisition time 3:02 min; coronal T1w: TR $550 \mathrm{~ms}$, TE $10 \mathrm{~ms}$, slice thickness $2.5 \mathrm{~mm}$, FoV $120 \times 179 \mathrm{~mm}^{2}$, acquisition time 2:23 $\mathrm{min}$.

- -Axial T2w: TR $3000 \mathrm{~ms}$, TE $120 \mathrm{~ms}$, slice thickness $3 \mathrm{~mm}$, FoV $150 \times 169 \mathrm{~mm}^{2}$, acquisition time 4:36 min; coronal T2w: TR $3036 \mathrm{~ms}$, TE $120 \mathrm{~ms}$, slice thickness $2.5 \mathrm{~mm}$, FoV $120 \times 179 \mathrm{~mm}^{2}$, acquisition time 3:51 min.

The acquisition protocol was completed with volumetric T1w turbo fast echo sequences (TR $14 \mathrm{~ms}$, TE $6.5 \mathrm{~ms}$, slice thickness $1.1 \mathrm{~mm}$, FoV $256 \times 240 \mathrm{~mm}^{2}$, acquisition time $5: 17 \mathrm{~min})$.

\section{Diagnostic usefulness analysis}

Three investigators (two experienced neuroradiologists and one experienced otoneurosurgeon) independently evaluated the MR findings. A 4-point scale ( $0=$ completely unusable, 1 = visible but not suitable for diagnostic purposes due to artifact contamination, $2=$ contaminated by artifact but adequate for diagnostic purposes, $3=$ highquality image of the anatomic structure) was adopted to describe 14 intracranial structures: frontal lobe, parietal lobe, temporal lobe, occipital lobe, hypophysis, internal auditory canal, cochlea, semi-circular canals, vestibulum, brainstem, anterior lobe of the cerebellum, cerebellar vermis, middle cerebellar pedunculus and the cerebellopontine angle. Ipsilateral and contralateral structures, with respect to the CI side, were examined. When unpaired median structures were described (e.g., hypophysis, brainstem, cerebellar vermis), both ipsi- and contralateral sides of each structure were evaluated. Finally, MRI findings were analyzed to investigate specific questions:

a) Which CI position allows the best image quality score referring to a specific anatomical structure?

b) Which anatomical structure modifies its MRI visibility under bilateral cochlear placement according to each CI position?

c) Which CI position allows the best global visualization of the intracranial structures?

\section{Statistical analysis}

Statistical analyses were performed using the R software ( $\mathrm{R}$ version 3.1.3, R Development Core Team, R Foundation for Statistical Computing, Wien, Austria). Descriptive statistics were calculated for all groups (the mean, standard deviation, median, minimum and maximum values). A Kruskal Wallis test was applied to determine whether significant differences existed between the groups. Dunn's multiple comparisons test was used as a post-hoc evaluation. The limit for statistical significance for all statistical tests was predetermined at $p<0.05$. Diagnostic validity of each structure was described using the following ranges (Fig. 2):

o $0 \leq \mathrm{X}<1.5$ : not assessable (NA)

o $1.5 \leq \mathrm{X}<2.25$ : involved by artifact but assessable for diagnostic purposes (A)

o $\quad X \geq 2.25$ : high-quality (HQ)
Fig. 2 Graphical view of the diagnostic usefulness analysis

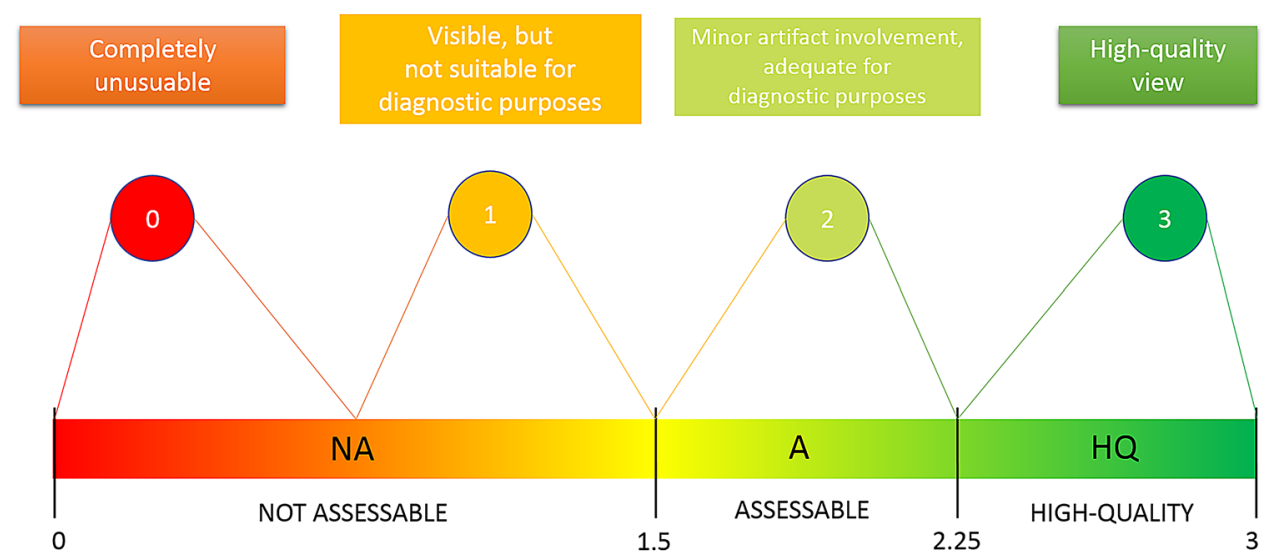


Inter-rater reliability was assessed using a Spearman $r$ correlation.

\section{Results}

Inter-rater reliability agreement ranged from 0.73 to 0.86 across all image evaluations and was consistent with "high positive correlation" among the three investigators. Volunteers reported no subjective discomfort (e.g., pressure on the side of the magnet, pain, magnet displacement) during the MRI examinations.

a) Which CI positioning allows the best image quality score referring to a specific anatomical structure?

When ipsilateral structures were examined under unilateral CI positioning, all 14 structures came out as assessable for diagnostic purposes in at least one CI position (Table 1). Moreover, view quality appeared highly assessable for 11 structures in at least one CI position. The occipital lobe achieved the lowest mean quality score (0) while the hypophysis scored the highest (3). When each anatomical structure was considered, CI positioning significantly modified $(p<0.05)$ the image quality scores as follows:

- frontal lobe: $90^{\circ}$ vs. $160^{\circ}$

- occipital lobe: $90^{\circ}$ vs. $120^{\circ} / 90^{\circ}$ vs. $135^{\circ} / 90^{\circ}$ vs $160^{\circ}$

- $\quad$ semi-circular canals: $90^{\circ}$ vs. $160^{\circ}$

- vestibulum: $90^{\circ}$ vs. $160^{\circ}$ (Fig. 3) anterior lobe cerebellum: $90^{\circ}$ vs. $120^{\circ} / 90^{\circ}$ vs. $135^{\circ} / 90^{\circ}$ vs. $160^{\circ}$

- cerebellar vermis: $90^{\circ}$ vs. $120^{\circ} / 90^{\circ}$ vs. $135^{\circ} / 90^{\circ}$ vs. $160^{\circ} / 120^{\circ}$ vs. $160^{\circ} / 135^{\circ}$ vs. $160^{\circ}$

- middle cerebellar peduncle: $90^{\circ}$ vs. $160^{\circ} / 120^{\circ}$ vs. $160^{\circ} / 135^{\circ}$ vs. $160^{\circ}$

Structures sided contralateral from the CI showed HQ values in all four placements (Table 2). Differences among alternative placements resulted statistically significant $(p<0.05)$ for the cerebellar vermis only $\left(90^{\circ} \mathrm{vs}\right.$. $\left.160^{\circ}\right)$.

b) Which anatomical structure modifies its MRI visibility under bilateral cochlear placement according to each CI position?

When each anatomical structure was analyzed under bilateral cochlear placement, CI positioning significantly modified $(p<0.05)$ the image quality rating as observed under unilateral CI. In addition to these findings, a further artifact was observed involving all anatomical structures close to the signal void/distortion area produced by each CI in case of bilateral cochlear placement. Consequently, when ipsilateral structures were compared under for both unilateral and bilateral cases, visibility was significantly modified $(p<0.05)$ for the following CI positions (Table 3; Fig. 4):

- $\quad 90^{\circ}$ : parietal lobe and brainstem.

- $\quad 120^{\circ}$ : semi-circular canals, vestibulum, brainstem, anterior lobe of the cerebellum, cerebellar vermis, middle cerebellar peduncle.

Table 1 Anatomical visibility assessment: unilateral CI-ipsilateral structures

\begin{tabular}{|c|c|c|c|c|}
\hline \multirow[b]{2}{*}{ Ipsilateral structures } & \multicolumn{4}{|l|}{ Unilateral CI } \\
\hline & $90^{\circ}($ Mean-SD) & $120^{\circ}($ Mean-SD $)$ & $135^{\circ}($ Mean-SD $)$ & $160^{\circ}($ Mean-SD $)$ \\
\hline Frontal lobe & $\mathrm{A}(2.10-0.31)$ & A $(2.23-0.50)$ & HQ (2.33-0.48) & HQ (2.53-0.51) \\
\hline Parietal lobe & A $(1.70-0.60)$ & A $(1.67-0.71)$ & A $(1.80-0.41)$ & A $(1.73-0.58)$ \\
\hline Temporal lobe & A $(1.77-0.63)$ & A $(1.80-0.61)$ & A $(1.90-0.31)$ & $\mathrm{A}(2.00-0)$ \\
\hline Occipital lobe & A $(1.83-0.59)$ & NA $(0.33-0.48)$ & NA $(0.20-0.41)$ & NA $(0-0)$ \\
\hline Hypophysis & HQ (2.90-0.31) & HQ (3-0) & HQ (2.97-0.18) & HQ (3-0) \\
\hline Internal auditory canal & HQ (2.83-0.53) & HQ (2.63-0.77) & HQ (2.67-0.66) & HQ (2.77-0.63) \\
\hline Cochlea & HQ (2.83-0.53) & HQ (2.67-0.71) & HQ (2.70-0.65) & HQ (2.73-0.64) \\
\hline Semicircular canals & HQ (2.57-0.68) & HQ (2.47-0.82) & A $(2.17-0.80)$ & $\mathrm{A}(1.90-1.0)$ \\
\hline Vestibulum & HQ (2.73-0.64) & HQ (2.63-0.77) & HQ (2.47-0.82) & A $(2.17-1.02)$ \\
\hline Brainstem & HQ (2.90-0.31) & HQ (2.80-0.61) & HQ (2.80-0.48) & HQ (2.83-0.38) \\
\hline Anterior lobe of the cerebellum & HQ (2.63-0.77) & $\mathrm{A}(2.00-0.83)$ & $\mathrm{A}(1.87-0.73)$ & A $(1.67-0.66)$ \\
\hline Cerebellar vermis & HQ (2.80-0.61) & HQ (2.27-0.79) & $\mathrm{A}(2.23-0.73)$ & NA (1.47-0.73) \\
\hline Middle cerebellar peduncle & HQ (2.80-0.61) & HQ (2.70-0.65) & HQ (2.47-0.78) & A $(1.77-0.68)$ \\
\hline Cerebellopontine angle & HQ (2.77-0.57) & HQ (2.67-0.71) & HQ (2.67-0.71) & HQ (2.70-0.70) \\
\hline
\end{tabular}

$H Q$ high-quality image; A: image involved by artifact, but assessable for diagnostic purposes; $N A$ not assessable image; $S D$ standard deviation Mean and standard deviation referred to the image quality scores assessed by all investigators according to all MRI sequences for ipsilateral structures, with respect to the CI side 
Fig. 3 Ipsilateral inner ear and internal auditory canal MRI visibility according to each CI position on $2 \mathrm{D}$ axial $\mathrm{T} 2 \mathrm{w}$ sequences
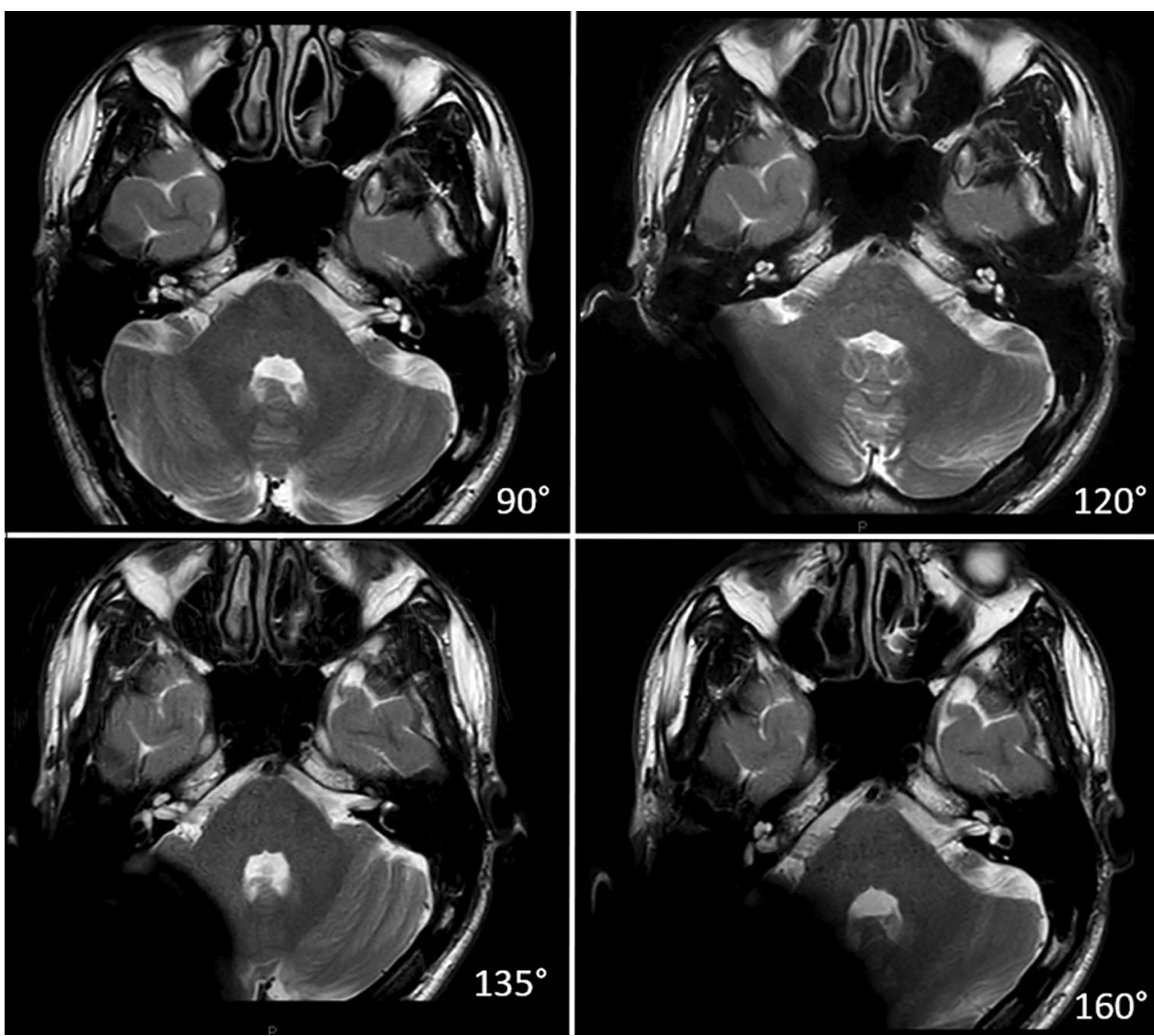

Table 2 Anatomical visibility assessment: unilateral CI-contralateral structures

\begin{tabular}{|c|c|c|c|c|}
\hline \multirow[t]{2}{*}{ Contralateral structures } & \multicolumn{4}{|l|}{ Unilateral CI } \\
\hline & $90^{\circ}$ (Mean-SD) & $120^{\circ}($ Mean-SD $)$ & $135^{\circ}($ Mean-SD $)$ & $160^{\circ}($ Mean-SD) \\
\hline Frontal lobe & HQ (2.80-0.40) & HQ (2.90-0.30) & HQ (2.90-0.30) & HQ (3-0) \\
\hline Parietal lobe & HQ (2.80-0.40) & HQ (2.90-0.30) & HQ (2.90-0.40) & HQ (2.90-0.30) \\
\hline Temporal lobe & HQ (2.90-0.30) & HQ (2.90-0.30) & HQ (3-0.2) & HQ (3-0) \\
\hline Occipital lobe & HQ (2.90-0.30) & HQ (2.70-0.70) & HQ (2.60-0.70) & HQ (2.60-0.60) \\
\hline Hypophysis & HQ (2.90-0.30) & HQ (3-0) & HQ (3-0.20) & HQ (3-0) \\
\hline Internal auditory canal & HQ (3-0.20) & HQ (3-0) & HQ (2.80-0.60) & HQ (3-0) \\
\hline Cochlea & HQ (3-0) & HQ (3-0) & HQ (2.80-0.60) & HQ (3-0) \\
\hline Semicircular canals & HQ (3-0) & HQ (3-0) & HQ (2.80-0.60) & HQ (3-0) \\
\hline Vestibulum & HQ (3-0.20) & HQ (3-0) & HQ (2.80-0.60) & HQ (3-0) \\
\hline Brainstem & HQ (2.90-0.30) & HQ (2.80-0.50) & HQ (2.90-0.40) & HQ (3-0) \\
\hline Anterior lobe of the cerebellum & HQ (3-0.20) & HQ (2.90-0.30) & HQ (2.80-0.60) & HQ (2.90-0.30) \\
\hline Cerebellar vermis & HQ (2.80-0.60) & HQ (2.70-0.70) & HQ (2.60-0.80) & HQ (2.40-0.90) \\
\hline Middle cerebellar peduncle & HQ (2.90-0.30) & HQ (2.80-0.60) & HQ (2.80-0.60) & HQ (3-0) \\
\hline Cerebellopontine angle & HQ (3-0) & HQ (3-0) & HQ (2.90-0.40) & HQ (3-0) \\
\hline
\end{tabular}

$H Q$ high-quality image; A: image involved by artifact, but assessable for diagnostic purposes; $N A$ not assessable image; $S D$ standard deviation Mean and standard deviation referred to the image quality scores assessed by all investigators according to all MRI sequences for contralateral structures, with respect to the CI side 


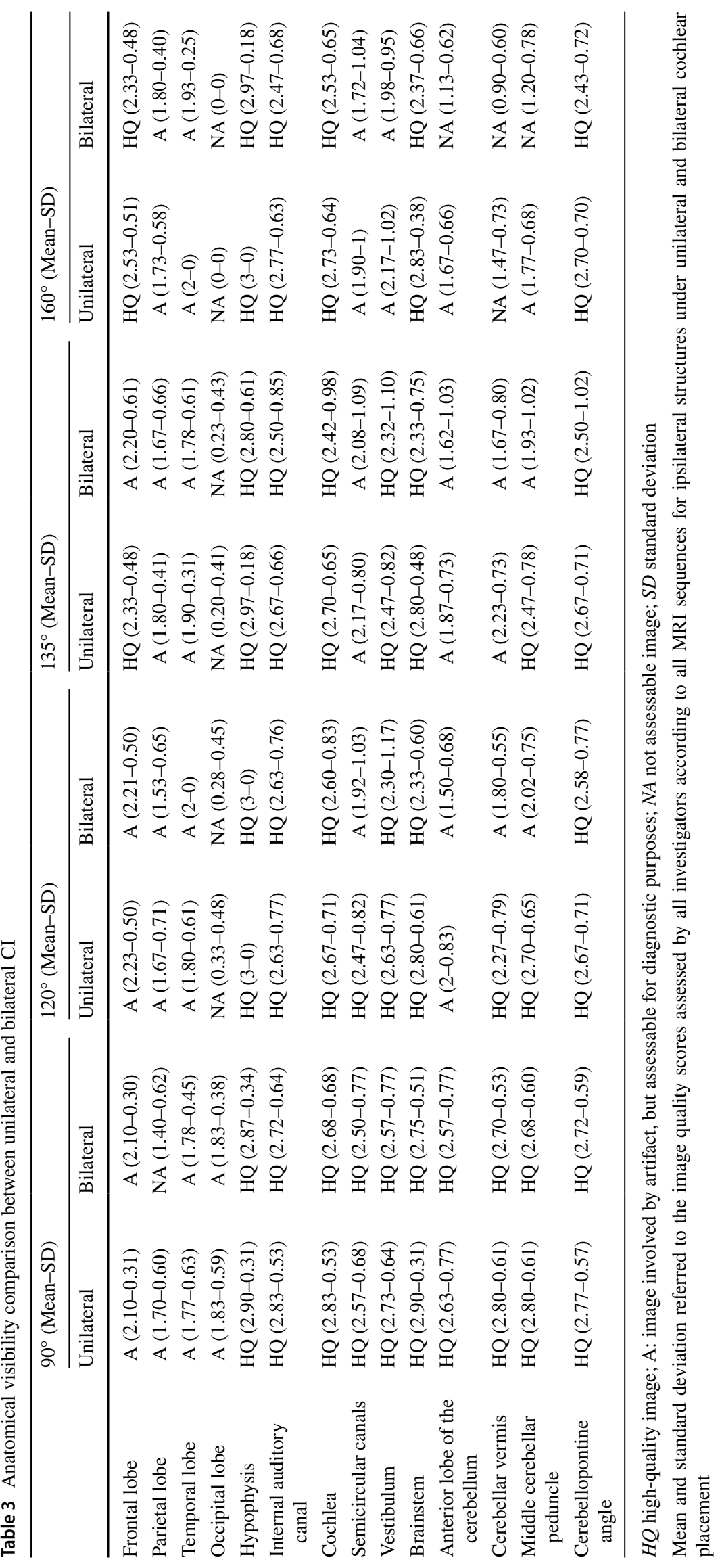


Fig. 4 Cerebellar vermis visibility according to $90^{\circ}$ and $120^{\circ}$ unilateral and bilateral CI positions. When $90^{\circ} \mathrm{CI}$ positioning was evaluated, the cerebellar vermis appeared highly visible for both unilateral and bilateral CI placement. When $120^{\circ} \mathrm{CI}$ positioning was considered, the cerebellar vermis visibility was highly assessable in unilateral cochlear placement; however, it was only assessable in case of bilateral CI placement
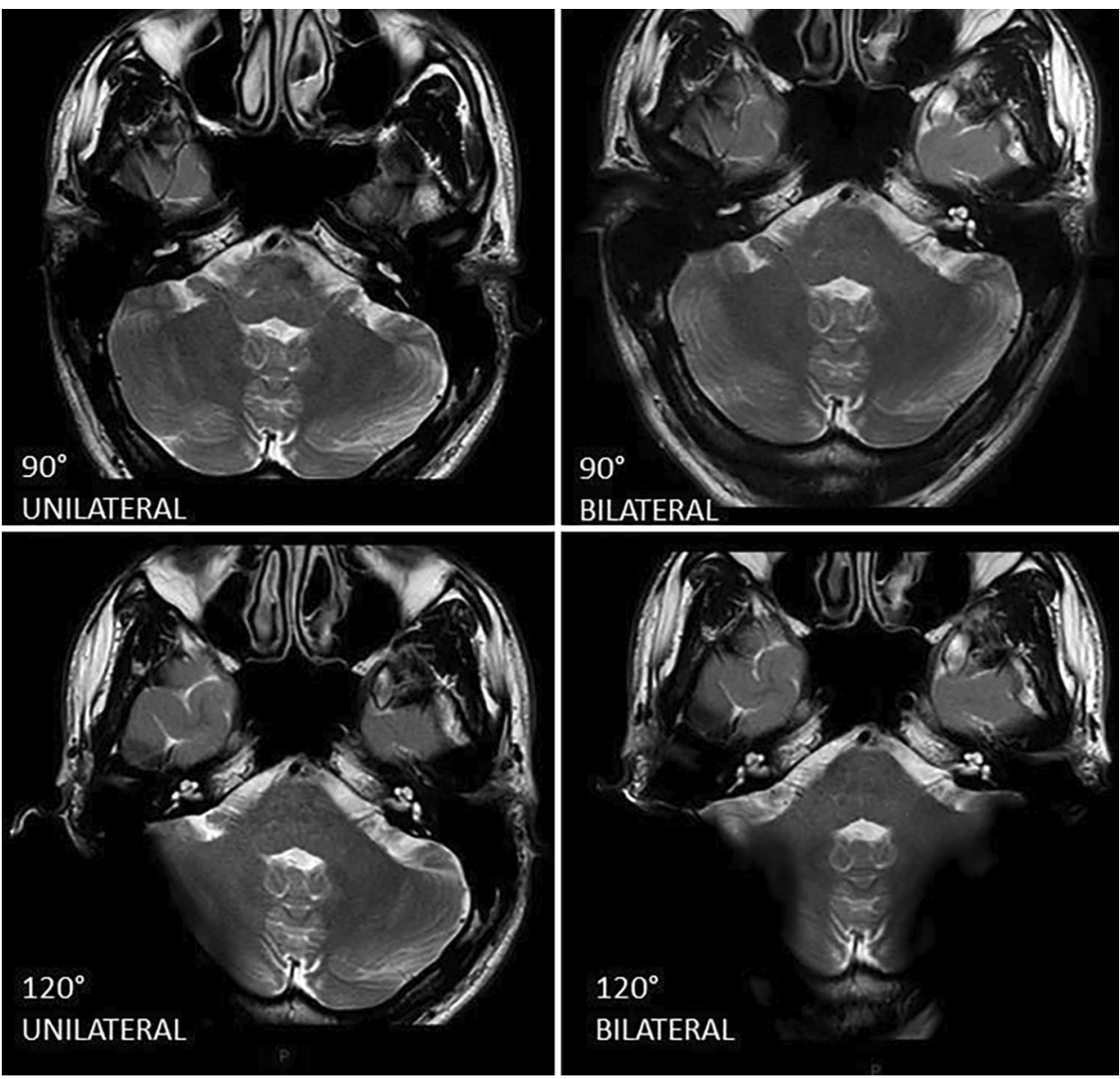

- $\quad 135^{\circ}$ : parietal lobe, cochlea, brainstem, cochlea, cerebellar vermis, middle cerebellar peduncle, cerebellopontine angle.

- $160^{\circ}$ : frontal lobe, internal auditory canal, brainstem, anterior lobe of the cerebellum, cerebellar vermis, middle cerebellar peduncle, cerebellopontine angle.

No statistically significant differences were found when the left and right structures were compared $(p>0.05)$ in case of bilateral cochlear placement.
An MRI diagnostic usefulness atlas considering cochlear implant positioning is supplied as supplementary material.

c) Which CI positioning allows the best global visualization of the intracranial structures?

When all 14 intracranial structures ipsilateral to cochlear implantation were globally evaluated, $90^{\circ}$ and $120^{\circ} \mathrm{CI}$ positioning allowed better image quality scores than $135^{\circ}(p>0.05)$ and $160^{\circ} \mathrm{CI}$ placement $(p<0.05$; Table 4$)$. No statistical differences were found when
Table 4 Global anatomical visibility assessment according to each CI positioning

\begin{tabular}{lllr}
\hline & Ipsilateral (Mean-SD) & Contralateral (Mean-SD) & Bilateral (Mean-SD) \\
\hline $90^{\circ}$ & HQ $(2.51-0.71)$ & HQ $(2.92-0.30)$ & HQ $(2.42-0.73)$ \\
$120^{\circ}$ & HQ $(2.28-0.93)$ & HQ $(2.90-0.38)$ & A $(2.06-0.95)$ \\
$135^{\circ}$ & A $(2.23-0.90)$ & HQ $(2.82-0.54)$ & A $(2.00-1.04)$ \\
$160^{\circ}$ & A $(2.09-0.98)$ & HQ $(2.91-0.34)$ & A $(1.84-0.99)$ \\
\hline
\end{tabular}

$H Q$ high-quality image; A: image involved by artifact, but assessable for diagnostic purposes; $N A$ not assessable image; $S D$ standard deviation

Mean and standard deviation referred to the image quality scores assessed by all investigators according to all intracranial structures and all MRI sequences under unilateral and bilateral cochlear placement 


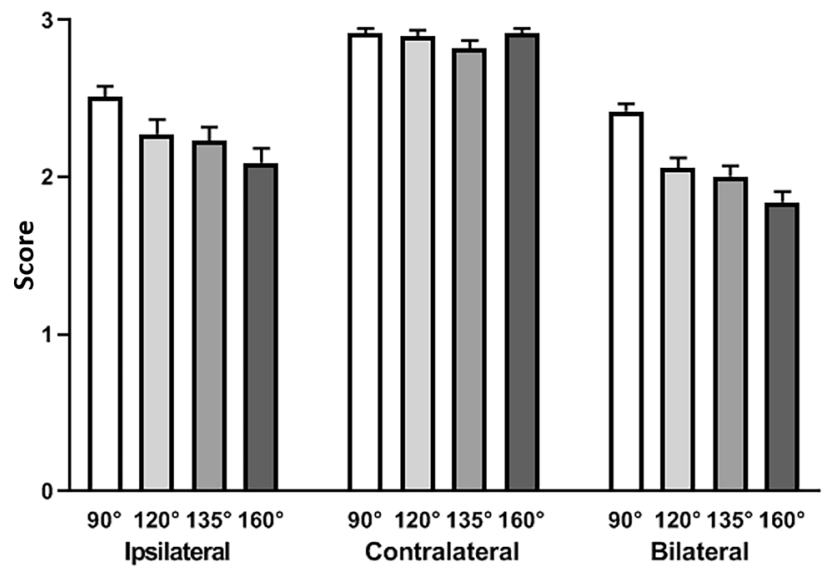

Fig. 5 Global anatomical visibility assessment. Global mean scores and confidence intervals according to each CI position and condition: unilateral (ipsilateral and contralateral structures visibility), bilateral cochlear placement

contralateral structures were analyzed according to each CI position $(p>0.05)$. In case of bilateral CI, surgical positioning significantly modified the image quality ratings as follows $(p<0.05)$ : $90^{\circ}$ vs. $120^{\circ} ; 90^{\circ}$ vs. $135^{\circ}$; $90^{\circ}$ vs. $160^{\circ} ; 120^{\circ}$ vs. $160^{\circ} ; 135^{\circ}$ vs. $160^{\circ}$. Overall, the $90^{\circ} \mathrm{CI}$ placement produced the best global image quality rating ( $p<0.05$; Fig. 5).

\section{Discussion}

The increasing number of patients undergoing CI surgery, related to an evident expansion of selection criteria [11-14] and an impressive evolution of health care technology [15, 16], has raised many concerns about the issue of CI-MRI compatibility $[17,18]$. How best to manage the MRI artifact represents one of the emerging topics related to the new generation of CIs. In the current study, the role of CI position on MR image quality was investigated to describe, for the first time, 14 intracranial structures following unilateral and bilateral CI placement. To more accurately simulate the MRinduced artifacts related to both the metallic CI components and the internal magnet system, we employed two Ultra 3D CIs. A paucity of work deals with MRI artifacts, focused on a few structures' visibility and involving CI dummies with nonrotatable magnets $[9,10]$. In our study, the diagnostic usefulness analysis was provided by three independent observers: with the high positive correlation between them supporting the reliability of the research. Previous studies found it difficult to rate visibility of anatomical structures for $3 \mathrm{~T}$ MRI scanning and for a CI distance lower than $9 \mathrm{~cm}$ from the outer-ear canal [7, 9, 10]. Moreover, such closer CI positions may lead to uncomfortable use of the "behind-the-ear" sound processor. Accordingly, four rotational CI orientations, each at $9 \mathrm{~cm}$ from the outer-ear canal, were investigated in relation to a specific anatomical structure. Each structure analyzed was of diagnostic interest and investigated using a 1.5 T MRI. The results demonstrated that each ipsilateral structure was sufficiently visible for diagnostic purposes in at least one CI position. At $90^{\circ}$ orientation, artifacts mainly involved brain structures located cranially and anteriorly (e.g., frontal lobe, temporal lobe); on the contrary, for the $160^{\circ}$ orientation, artifacts mostly influenced the MRI view of posterior fossa structures (e.g., occipital lobe, cerebellar vermis). More specifically, when the ipsilateral internal auditory canal and the cochlea were considered, they appeared highly visible for diagnostic purposes in all CI orientations. However, the ipsilateral vestibulum and semi-circular canals showed HQ image scores at $90^{\circ}$ and $120^{\circ}$ but received mainly assessable image quality ratings at $135^{\circ}$ and $160^{\circ}$ through being located more posteriorly. These findings were consistent with Todt et al. and Schröeder et al. [9, 10], even if the authors did not make a comparison between each inner ear structure. Interestingly, Todt et al. [9] observed one magnet dislocation and described candidates' painful pressure during $3 \mathrm{~T} \mathrm{MRI}$; Schröeder et al. [10] reported volunteers' pressure sensations on the side of the magnet during $1.5 \mathrm{~T}$ MRI. In contrast, we did not experience any degree of subjective discomfort, in agreement with previous studies involving adaptive magnets [19-22]. The role of CI position on MRI artifact was also investigated for bilateral CI placement. In this condition, MR imaging revealed additional artifacts involving all structures located close to the signal void/distortion area produced by each CI. Posterior fossa structures worsened in their visibility at $160^{\circ}$ bilateral $\mathrm{CI}$ positioning in comparison with unilateral CI. On the contrary, anterior, and cranial brain structures were more involved by additional artifacts at a $90^{\circ}$ bilateral CI position. Overall, the 90 -degree orientation provided the best visibility rating concerning the brain structures analyzed, which mainly belonged to the posterior cranial fossa. However, a 90-degree CI position could face daily life problems when wearing caps or headbands, suggesting that in these cases a personalized solution is found. Previous authors have looked at different strategies to handle the impact of MRI artifacts on image quality. Wackym and colleagues [4] found that head rotation angle along the $\mathrm{z}$-axis influences the image degradation produced by the internal non-rotatable magnet. Ay et al. [5] showed that an anteflexion of the head inside the MRI scanner improves the visualization in the coronal plane of the inner auditory canal. Wagner et al. [3] studied the positive effects of magnet removal on image quality; however, a risk-benefits analysis should be carefully pondered concerning the drawbacks of revision surgery. Sharon et al. [6] published the results of acquisition imaging techniques aimed at formulating an MRI protocol to improve image visibility. Finally, it was recently demonstrated how different 
neurological disorders may require different strategies aimed at MRI follow-up, highlighting the role of pre-operative planning [17]. Some critical considerations should be applied to our findings. Despite the highly positive correlation among the three observers, our findings were limited to observations made on two adult male volunteers: further data based on skull sizes of different dimensions (e.g., pediatric ones) should be contemplated. The unavailability of a 3 T MRI scanner at our institution limited our results to $1.5 \mathrm{~T}$ MRIinduced artifacts. A comparison among MRI artifacts produced by CI models with different diametrically bipolar magnets (e.g., Med-El Synchrony; Cochlear Nucleus Profile Plus) should be considered by further studies. When surgically implanted CI devices are investigated, MR imaging quality might show some differences compared to our external skinsurface position. Finally, additional artifacts produced by the intracochlear electrode array insertion should be further considered.

\section{Conclusions}

The introduction of adaptive CI magnet systems has created a new scenario in the world of CI recipients. The need for post-surgical MRI surveillance may be overcome thanks to the adoption of strategies aimed at handling the MRIinduced artifacts. This study found that careful CI positioning allows for the visualization of various intracranial structures with sufficient visualization for diagnostic purposes, in case of unilateral CI. Bilateral CIs significantly deteriorate the anatomical visibility because of the two implant magnets' mutual interaction. What structures need to be viewed? Which MRI sequences are required? Answering these questions should now be part of pre-operative CI planning. CI positioning may play a crucial role in guiding the best surgical choice. However, further studies are mandatory to support and extend our research.

Supplementary Information The online version contains supplementary material available at https://doi.org/10.1007/s00405-021-07005-y.

Funding Open access funding provided by Università degli Studi di Pavia within the CRUI-CARE Agreement. The authors of this manuscript declare that they thank the Advance Bionics AG [Advanced Bionics, Stäfa, Switzerland] for loan of the cochlear implants and funding that study [total amount $32,000 €$ ]. The authors state that this work has received funding from Advance Bionics AG [Advanced Bionics, Stäfa, Switzerland] in the amount of 32,000€. Additionally, the authors confirm that the funders had no role in our study design, data collection and analysis, decision to publish, or preparation of the manuscript.

\section{Declarations}

Conflict of interests The authors of this manuscript declare that they thank the Advance Bionics AG [Advanced Bionics, Stäfa, Switzerland] for loan of the cochlear implants and funding that study [total amount $32,000 €]$. The authors state that this work has received funding from Advance Bionics AG [Advanced Bionics, Stäfa, Switzerland] in the amount of 32,000€. This does not alter the authors' adherence to Eur Arch Otorhinolaryngol. policies on sharing data and materials.

Ethical approval This study was reviewed and approved by the institutional review board and medical ethics committee of the IRCCS Policlinico San Matteo, Pavia (IRB\# 20210027855). Volunteers offered written informed consent.

Open Access This article is licensed under a Creative Commons Attribution 4.0 International License, which permits use, sharing, adaptation, distribution and reproduction in any medium or format, as long as you give appropriate credit to the original author(s) and the source, provide a link to the Creative Commons licence, and indicate if changes were made. The images or other third party material in this article are included in the article's Creative Commons licence, unless indicated otherwise in a credit line to the material. If material is not included in the article's Creative Commons licence and your intended use is not permitted by statutory regulation or exceeds the permitted use, you will need to obtain permission directly from the copyright holder. To view a copy of this licence, visit http://creativecommons.org/licenses/by/4.0/.

\section{References}

1. Shew M, Wichova H, Lin J, Ledbetter LN, Staecker H (2019) Magnetic resonance imaging with cochlear implants and auditory brainstem implants: are we truly practicing MRI safety? Laryngoscope 129:482-489. https://doi.org/10.1002/lary.27516

2. MRI safety information for the HiRes Ultra 3D cochlear implant (2018) In: LLC AB, ed.; 1-5

3. Wagner F, Wimmer W, Leidolt L, Vischer M, Weder S, Wiest R, Mantokoudis G, Caversaccio MD (2015) Significant artifact reduction at $15 \mathrm{~T}$ and $3 \mathrm{~T}$ MRI by the use of a cochlear implant with removable magnet: an experimental human cadaver study. PLoS ONE 10:e0132483. https://doi.org/10.1371/journal.pone. 0132483

4. Wackym PA, Michel MA, Prost RW, Banks KL, Runge-Samuelson CL, Firszt JB (2004) Effect of magnetic resonance imaging on internal magnet strength in Med-El Combi $40+$ cochlear implants. Laryngoscope 114:1355-1361. https://doi.org/10.1097/00005537200408000-00007

5. Ay N, Gehl HB, Sudhoff H, Todt I (2020) Effect of head position on cochlear implant MRI artifact. Eur Arch Otorhinolaryngol. https://doi.org/10.1007/s00405-020-06349-1 (online ahead of print)

6. Sharon JD, Northcutt BG, Aygun N, Francis HW (2016) Magnetic resonance imaging at 1.5 tesla with a cochlear implant magnet in place: image quality and usability. Otol Neurotol 37:1284-1290. https://doi.org/10.1097/MAO.0000000000001176

7. Majdani O, Rau TS, Götz F, Zimmerling M, Lenarz M, Lenarz T, Labadie R, Leinung M (2009) Artifacts caused by cochlear implants with non-removable magnets in 3T MRI: phantom and cadaveric studies. Eur Arch Otorhinolaryngol 266:1885-1890. https://doi.org/10.1007/s00405-009-0994-8

8. Canzi P, Aprile F, Simoncelli A, Manfrin M, Magnetto M, Lafe E, Minervini D, Avato I, Terrani S, Scribante A, Gazibegovic D, Benazzo M (2020) MRI-induced artifact by a cochlear implant 
with a novel magnet system: an experimental cadaver study. Eur Arch Otorhinolaryngol. https://doi.org/10.1007/s00405-02006464-z (online ahead of print)

9. Todt I, Rademacher G, Mittmann P, Wagner J, Mutze S, Ernst A (2015) MRI artifacts and cochlear implant positioning at 3T in vivo. Otol Neurotol 36:972-976. https://doi.org/10.1097/MAO. 0000000000000720

10. Schröder D, Grupe G, Rademacher G, Mutze S, Ernst A, Seidl R, Mittmann P (2018) Magnetic resonance imaging artifacts and cochlear implant positioning at $1.5 \mathrm{~T}$ in vivo. Biomed Res Int 2018:9163285. https://doi.org/10.1155/2018/9163285

11. Carlson ML, Sladen DP, Haynes DS, Driscoll CL, DeJong MD, Erickson HC, Sunderhaus LW, Hedley-Williams A, Rosenzweig EA, Davis TJ, Gifford RH (2015) Evidence for the expansion of pediatric cochlear implant candidacy. Otol Neurotol 36:43-50. https://doi.org/10.1097/MAO.0000000000000607

12. Gifford RH, Dorman MF, Shallop JK, Sydlowski SA (2010) Evidence for the expansion of adult cochlear implant candidacy. Ear Hear 31:186-194. https://doi.org/10.1097/AUD.0b013e3181 c6b831

13. Canzi P, Manfrin M, Perotti M, Aprile F, Quaglieri S, Rebecchi E, Locatelli G, Benazzo M (2017) Translabyrinthine vestibular neurectomy and simultaneous cochlear implant for Ménière's disease. Acta Neurochir (Wien) 159:123-130. https://doi.org/10. 1007/s00701-016-2996-9

14. Canzi P, Pecci A, Manfrin M, Rebecchi E, Zaninetti C, Bozzi V, Benazzo M (2016) Severe to profound deafness may be associated with MYH9-related disease report of 4 patients. Acta Otorhinolaryngol Ital 36:415-420. https://doi.org/10.14639/ 0392-100X-702

15. Lenarz T (2018) Cochlear implant - state of the art. GMS Curr Top Otorhinolaryngol Head Neck Surg 16:Doc04. https://doi.org/ 10.3205/cto000143

16. Edmonson HA, Carlson ML, Patton AC, Watson RE (2018) MR Imaging and cochlear implants with retained internal magnets: reducing artifacts near highly inhomogeneous magnetic fields. Radiographics 38:94-106. https://doi.org/10.1148/rg.2018170135

17. Barbara M, Margani V, Costanzo R, Salvetti M, Bozzao A, Monini S, Covelli E (2021) Cochlear implantation in neurological patients: case report and literature survey. Laryngoscope 131:E576-E580. https://doi.org/10.1002/lary.28704

18. Sudhoff H, Gehl HB, Scholtz LU, Todt I (2020) MRI observation after intralabyrinthine and vestibular schwannoma resection and cochlear implantation. Front Neurol 11:759. https://doi.org/10. 3389/fneur.2020.00759

19. Todt I, Tittel A, Ernst A, Mittmann P, Mutze S (2017) Pain free 3 T MRI scans in cochlear implantees. Otol Neurotol 38:e401-e404. https://doi.org/10.1097/MAO.0000000000001569

20. Todt I, Rademacher G, Grupe G, Stratmann A, Ernst A, Mutze S, Mittmann P (2018) Cochlear implants and 15 T MRI scans: the effect of diametrically bipolar magnets and screw fixation on pain. J Otolaryngol Head Neck Surg 47:11. https://doi.org/10. 1186/s40463-017-0252-9

21. Cass ND, Honce JM, O’Dell AL, Gubbels SP (2019) First MRI with new cochlear implant with rotatable internal magnet system and proposal for standardization of reporting magnet-related artifact size. Otol Neurotol 40:883-891. https://doi.org/10.1097/ MAO.0000000000002269

22. Tysome JR, Tam YC, Patterson I, Graves MJ, Gazibegovic D (2019) Assessment of a novel 3T MRI compatible cochlear implant magnet: torque, forces, demagnetization, and imaging. Otol Neurotol 40:e966-e974. https://doi.org/10.1097/MAO.00000 00000002407

Publisher's Note Springer Nature remains neutral with regard to jurisdictional claims in published maps and institutional affiliations. 